\title{
The differential outcomes effect in pigeons is not reduced by eliminating response-outcome associations: Support for a two-process account
}

\author{
LOU M. SHERBURNE \\ Wabash College, Crawfordsville, Indiana \\ and \\ THOMAS R. ZENTALI \\ University of Kentucky, Lexington, Kentucky
}

\begin{abstract}
Animals perform two-choice conditional discriminations at a higher level if each of the two correct responses to the comparison stimuli is reinforced with a different outcome. According to the two-process view, this differential outcomes effect (DOE) results from the response-cuing function of expectancies generated by the conditional stimuli (i.e., samples). According to the shared-outcomes view, differential response-outcome associations contribute to the effect. In the present research, pigeons that were trained with differential outcomes associated with the samples, but not with the comparisons, revealed a DOE during delay testing that was comparable to that obtained in a "true" differential-outcomes group. Thus, a two-process interpretation of the DOE was supported. In the second experiment, transfer testing with sample replacement confirmed these findings and, in addition, provided evidence that differential sample behaviors exerted some control over zero-delayed choice.
\end{abstract}

In a typical differential-outcomes conditional discrimination with pigeons, a correct response following one of two conditional stimuli (e.g., R1, after Sample 1, S1) is reinforced with one outcome (O1, e.g., food) and a correct response following the other conditional stimulus (e.g., R2, after Sample 2, S2) is reinforced with a different outcome (O2, e.g., water). Pigeons trained with such a procedure often acquire the task more rapidly and perform it at a higher level than pigeons in a control group in which correct responses are reinforced with outcomes that are uncorrelated with the samples. This differential outcomes effect (DOE) is especially apparent when correct performance is made more difficult by delaying presentation of the comparisons (see, e.g., Peterson, Wheeler, $\&$ Trapold, 1980). Similar results have been reported using differential delay (with rats, Carlson \& Wielkiewicz, 1972), differential magnitude (e.g., with rats, Carlson \& Wielkiewicz, 1976), and differential probability (e.g., with pigeons, DeMarse \& Urcuioli, 1993; Urcuioli, 1990) of the same reinforcer.

This research was supported by a University of Kentucky Dissertation Fellowship awarded to L.M.S. and by National Institute of Mental Health Grant MH 45979 and National Science Foundation Grant BNS 9019080, awarded to T.R.Z. We thank Karen Roper, Daren Kaiser, Jorga Weaver, and Brigette Cook for their assistance with data collection. Some of these data were presented at the 35 th annual meeting of the Psychonomic Society, St. Louis (November 1994). Correspondence should be addressed to L. M. Sherburne, Department of Psychology, Wabash College, Crawfordsville, IN 47933 (e-mail address: sherburl@scholar.wabash.edu).
The results of transfer-of-control experiments have helped to identify the processes underlying the DOE. In one of these experiments, for example, Peterson (1984) trained pigeons on a conditional discrimination task with food-versus-tone (i.e., food vs. the absence of food) differential outcomes. Off baseline, the pigeons were trained on a simple successive discrimination in which new stimuli were differentially associated with the outcomes. On a transfer test in which the initial stimuli from the conditional discrimination were replaced with those from the simple discrimination, the pigeons showed excellent transfer of performance.

These and other similar transfer results rule out a number of possible interpretations of the DOE. Enhanced discriminability of sample or comparison stimuli, for example, might reasonably account for differential-outcomes acquisition and performance effects, but cannot account for the transfer results. Instead, the substitutability of stimuli that share outcome associations strongly suggests that differential-outcomes performance is mediated by differential-outcome expectancies. The nature of such expectancy mediation has been disputed, however.

Perhaps the simplest of the expectancy-mediation accounts of the DOE is that based on the differential sample behaviors that result from differential-outcome associations (Jenkins \& Moore, 1973). When the differential outcomes are food versus the absence of food (e.g., feeder light), for example, pigeons typically peck the foodassociated sample but do not peck the no-food-associated sample. Urcuioli and DeMarse (1994) have, in fact, demonstrated that the differential sample behaviors that re- 
sult from training with food-versus-light differential outcomes do provide cues sufficient to produce a differentialoutcomes transfer effect. And when differential sample responding has been eliminated, differential-outcomes acquisition effects have been diminished or eliminated (Zentall \& Sherburne, 1994). Control of differential sample responding does not eliminate superior differentialoutcomes delay performance and transfer effects, however (Delong \& Wasserman, 1981; Fedorchak \& Bolles, 1986; Sherburne \& Zentall, 1995; Zentall \& Sherburne, 1994). Thus, outcome expectancies themselves, and not simply the external behaviors they produce, apparently can exert control over comparison responses in a differentialoutcomes task.

Two models of the associative processes involved in such control by expectancies have been proposed. On the one hand, Trapold (1970) and others (e.g., Overmier \& Lawry, 1979; Peterson, 1984; Trapold \& Overmier, 1972) have argued that an outcome expectancy is first established and then comes to be associated with whichever of the two comparison responses is reinforced in its presence. According to this two-process interpretation, the Pavlovian sample-outcome associations that produce differential sample-generated expectancies are established before the differential-outcomes conditional discrimination is learned. Such expectancies provide cues that are unavailable to animals trained with a nondifferentialoutcomes task - cues that facilitate acquisition by providing additional discriminative stimuli for association with correct-comparison responses. Once the conditional discrimination is learned, the differential expectancies also provide an additional source of cues for mediating correct performance when responding to the comparisons is delayed.

In a differential-outcomes procedure, differential Pavlovian associations are likely to develop not only between the sample stimuli and the outcomes that follow them, but also between the comparison stimuli and those outcomes. However, in the two-process view, any differential expectancies that become associated with the comparison stimuli (or with responses to them) play no direct role in the DOE. Reinforcement of a correct-comparison response serves only to strengthen the association between a sample-generated expectancy (i.e., the discriminative stimulus) and choice of the appropriate comparison (i.e., the instrumental response).

On the other hand, Rescorla (1992) has suggested that the DOE provides evidence of the response-outcome associations that some theorists have viewed as being critical to instrumental performance (see Colwill \& Rescorla, 1985). According to this interpretation, the DOE results because one of the samples (S1) and a response to one of the comparisons ( $\mathrm{R} 1)$ share an association with one of the outcomes (O1), while the other sample (S2) and a response to the other comparison (R2) share an association with the other outcome $(\mathrm{O} 2)$. When, for example, an expectancy of $\mathrm{O} 1$ is generated by $\mathrm{S} 1$, the $\mathrm{O} 1$ expectancy produces R1 through a backward association. Similarly, an expectancy of $\mathrm{O} 2$ generated by $\mathrm{S} 2$ results in $\mathrm{R} 2$. In other words, it is the match between the outcome expectancies generated by $\mathrm{S} 1$ and $\mathrm{R} 1$ (and between $\mathrm{S} 2$ and $\mathrm{R} 2$ ) that produces a DOE.

Thus, according to the shared-outcomes interpretation, animals need not learn to use outcome expectancies as discriminative stimuli for instrumental responses. Instead, differential outcomes enhance conditional discrimination performance because correct-comparison responses are elicited by sample-generated representations of the outcomes associated with those responses. The two-process and shared-outcomes interpretations of the DOE are illustrated in Table 1.

A great deal of support for the two-process interpretation has been generated experimentally over the last two decades. Transfer-of-control results, in particular, have been viewed as evidence that, during differential-outcomes training, animals learn to use sample-produced outcome expectancies as cues for comparison responses. The majority of these findings, however, also lend themselves to a shared-outcomes interpretation. It could be argued that sample-produced outcome expectancies differentially elicit comparison responses that are, themselves, associated with the expected outcomes.

Recently, Urcuioli and DeMarse (1996) have attempted to distinguish between the two interpretations by asking whether or not the DOE is dependent on pigeons having learned to use sample-generated expectancies as choice cues. They first trained pigeons on two conditional discriminations that involved the same samples ( $\mathrm{S} 1$ and $\mathrm{S} 2$ ), but different comparison responses (R1 and R2 on some trials; R3 and R4 on the others). By using this oneto-many (OTM) sample-to-comparison mapping, it was possible to manipulate differential sample-outcome associations while holding differential response-outcome associations constant. In the critical group, the pigeons were trained, during each session, with S1-R1-O1, S1$\mathrm{R} 3-\mathrm{O} 2, \mathrm{~S} 2-\mathrm{R} 2-\mathrm{O} 2$, and $\mathrm{S} 2-\mathrm{R} 4-\mathrm{O} 1$ associations. Because each of the samples was associated equally with each of the outcomes, there was no opportunity for the pigeons to develop differential expectancies during sample presentations. When these samples were then replaced by stimuli from a simple discrimination (S3 and S4) in which the stimuli had been differentially associ-

Table 1

Presumed Associations Involved in a Standard Differential Outcomes Procedure

\begin{tabular}{ccc}
\hline & \multicolumn{2}{c}{ Presumed Associations } \\
\cline { 2 - 3 } Training & Two Process & Shared Outcome \\
\hline S1 trials: $\mathrm{S} 1-\mathrm{R} 1-\mathrm{O} 1$ & $\mathrm{~S} 1 \rightarrow$ expect $\mathrm{O} 1$ & $\mathrm{~S} 1 \rightarrow \operatorname{expect} \mathrm{O} 1$ \\
& expect $\mathrm{O} 1 \rightarrow \mathrm{R} 1$ & $\mathrm{R} 1 \leftarrow$ expect $\mathrm{O} 1$ \\
S2 trials: $\mathrm{S} 2-\mathrm{R} 2-\mathrm{O} 2$ & $\mathrm{~S} 2 \rightarrow$ expect $\mathrm{O} 2$ & $\mathrm{~S} 2 \rightarrow$ expect $\mathrm{O} 2$ \\
& expect $\mathrm{O} 2 \rightarrow \mathrm{R} 2$ & $\mathrm{R} 2 \leftarrow$ expect $\mathrm{O} 2$ \\
\hline
\end{tabular}

Note - $S$, initial stimulus; $R$, comparison response reinforced; $O$, outcome used to reinforce a correct response. 
ated with the two outcomes ( $33-\mathrm{O} 1, \mathrm{~S} 4-\mathrm{O} 2)$, however, the birds reliably made the response that shared an outcome association with the test stimulus (i.e., they showed a DOE).

Urcuioli and DeMarse's (1996) results, together with similar transfer-of-control findings reported by Rescorla (1992, Experiment 2) with rats, suggest that the DOE does not result solely from animals learning to use differentialoutcome expectancies as choice cues. Instead, consistent with a shared-outcomes account of the DOE, these results suggest that an outcome expectancy "naturally" elicits a response that is, itself, associated with that outcome.

Alternatively, it is possible that the procedures used in these experiments did not actually prevent the animals from learning to use expectancies as cues. Although, in Urcuioli and DeMarse's (1996) experiment, the samples alone did not provide the basis for differential-outcome expectancies, comparison presentation combined with sample memory may have provided such a basis. We have, in fact, relied on such serial-compound cues as the basis for the development of differential-outcome expectancies in previous research. To prevent use of differentialsample-responding cues, we used a differential-outcomes procedure in which responding (fixed ratio [FR], 10) was required to both samples (Sherburne \& Zentall, 1995; Zentall \& Sherburne, 1994). In these experiments, a correct-comparison response (Rl) after one of the samples (S1) led to food access (O1). A correct-comparison response (R2) after the other sample (S2) led to the absence of food ( $\mathrm{O} 2$; feeder light only). The birds were persuaded to peck $\mathrm{S} 2$ by including occasional (and unpredictable) trials on which responding to $\mathrm{S} 2$ resulted in presentation of food $(\mathrm{O} 1)$, rather than presentation of the comparisons. Thus, on any $\mathrm{S} 2$ trial, an expectation of $\mathrm{O} 2$ for correct-comparison response could not be established until the comparisons were presented. We argued that the functional cue for producing an $\mathrm{O} 2$ expectancy on such choice trials was a serial compound: S2 followed by comparison presentation (or, alternatively, S2 not followed immediately by food). Similar processes could explain the transfer results reported by Urcuioli and DeMarse (1996) and by Rescorla (1992). For example, pigeons might learn to use the $\mathrm{O} 1$ expectancy generated by one serial compound ( $\mathrm{S} 1$ followed by presentation of the $\mathrm{R} 1$ and $\mathrm{R} 2 \mathrm{com}$ parisons) as a cue for $\mathrm{R} 1$, and the $\mathrm{O} 2$ expectancy generated by a different serial compound ( $\mathrm{S} 3$ followed by presentation of the $\mathrm{R} 1$ and $\mathrm{R} 2$ comparisons), as a cue for R2. Thus, the results of transfer-of-control experiments employing an OTM procedure could be interpreted reasonably in terms of either a two-process account that incorporates the use of serial-compound expectancy cues or a shared-outcomes account.

An alternative approach to an empirical distinction between the two hypotheses is to manipulate the responseoutcome associations required by the shared-outcomes account, rather than manipulating the prerequisite learning involved in the two-process account. According to the shared-outcomes model, it is important that different out- comes be associated with the two comparison responses. Nondifferential reinforcement of correct-comparison responses should decrease the magnitude of the DOE because the outcome expectancies produced by the sample stimuli should not differentially elicit comparison responses. According to the two-process model, however, different outcomes need not be associated with the two comparison responses. Instead, as long as differential sample-outcome associations are maintained, nondifferential reinforcement of correct-comparison responses should not decrease the magnitude of the DOE.

In the typical differential-outcomes procedure used with pigeons, it is not possible to manipulate whether or not differential outcomes are associated with the two comparison responses while holding differential sample outcome associations constant. Expectancies established during sample presentations are perfectly correlated with the outcomes obtained for correct-comparison responses. In the present research, however, this correlation was eliminated in the critical group (the sample outcome [SO] group) by using a variant of the OTM procedure to establish differential sample-outcome expectancies on trials during which a comparison response was not required.

On most trials, either S1 was followed immediately by $\mathrm{O} 1$ or $\mathrm{S} 2$ was followed immediately by $\mathrm{O} 2$. On a relatively few interspersed trials, comparisons were presented and correct responses ( $\mathrm{R} 1$ after S1, R2 after S2) were reinforced with nondifferential outcomes. The design of this experiment is presented in Table 2. Because the "samples" that were to be followed immediately by an outcome were indistinguishable from those to be followed by comparisons, differential-outcome expectancies could be generated during the sample presentations on choice trials, despite the fact that correct-comparison responses were reinforced nondifferentially. Although,

Table 2 Design of Experiment 1

\begin{tabular}{lrcc}
\hline & \multicolumn{3}{c}{ Number of Trials of Each Trial Type $\times$ Group } \\
\cline { 2 - 4 } \multicolumn{1}{c}{ Trial Type } & SO & SCO & NDO \\
\hline S1-B1-O1 & 48 & 40 & 24 \\
S1-B1-O2 & 0 & 8 & 24 \\
S2-B2-O1 & 0 & 8 & 24 \\
S2-B2-O2 & 48 & 40 & 24 \\
S1-B1-R1-O1 & 12 & 20 & 12 \\
S1-B1-R1-O2 & 12 & 4 & 12 \\
S2-B2-R2-O1 & 12 & 4 & 12 \\
S2-B2-R2-O2 & 12 & 20 & 12 \\
\hline
\end{tabular}

Note- -S1, Sample 1 (red hue for half the birds, green hue for the others); S2, Sample 2 (green hue for half the birds, red for the others); $\mathrm{B} 1$, Behavior 1 (fixed ratio 10); B2, Behavior 2 (differential reinforcement of other behavior $6 \mathrm{sec}$ ); R 1, correct comparison Response 1 (choice of a vertical line); R2, correct comparison Response 2 (choice of a horizontal line); O1, Outcome 1 (food); O2, Outcome 2 (no food, feeder light). The conditional and simple discrimination trials were randomly interspersed throughout the session. Groups are designated by differential-outcome associations: $\mathrm{SO}$, samples, but not comparisons, were associated with differential outcomes; SCO, both samples and comparisons were associated with differential outcomes; NDO, neither samples nor comparisons were associated with differential outcomes. 
with this procedure, any differential expectancies established during the sample presentations should be disconfirmed by comparison presentation, memory for such expectancies should still be available at the time the comparison response is made.

According to the two-process account, such memories could be expected to serve as comparison-response cues and could also mediate delays, in much the same way that memories for the hue of a sample, or for sampledirected behavior, serve as cues in a nondifferentialoutcomes procedure. The degree to which expectancies serve more effectively as cues than sample hue or sample behavior should be reflected in superior performance relative to an appropriate nondifferential-outcomes control.

According to the shared-outcomes account, however, the performance of animals trained with such a task should be worse than the performance of animals trained with a task in which differential sample-generated expectancies are shared by the comparison responses with which they are associated (i.e., a standard differentialoutcomes task). The associations presumed to be involved in the SO group task, according to the two-process and the shared-outcomes accounts, are contrasted in Table 3 .

In Experiment 1, pigeons in the SO group were trained using the procedure outlined above. For these pigeons, although the samples were associated with differential probabilities of the two outcomes, comparison responses were not. For a second group of pigeons, both the samples and the comparison responses were associated with differential probabilities of the two outcomes (the samplecomparison-outcome [SCO] group; analogous to a standard differential-outcomes group). For a third group, neither the samples nor the comparison responses were associated with differential outcomes (the nondifferentialoutcome [NDO] control group).

Two secondary procedural issues should be clarified. First, because differential-sample responding is known to facilitate both acquisition (Urcuioli \& Honig, 1980) and delay performance (Zentall, Hogan, Howard, \& Moore, 1978) of a nondifferential-outcomes task, we held such responding constant across groups by requiring all pigeons to respond to one of the samples (FR 10) and to withhold responding to the other sample (differential reinforcement of other behavior, DRO, $6 \mathrm{sec}$ ). In previous studies, similar control of differential-sample responding has diminished (Zentall \& Sherburne, 1994; Experiment 2) or eliminated (Zentall \& Sherburne, 1994, Experiment 1) any

Table 3

Presumed Associations Involved in the SO Group Differential Outcomes Procedure

\begin{tabular}{cll}
\hline \multirow{2}{*}{ Training } & \multicolumn{2}{c}{ Presumed Associations } \\
\cline { 2 - 3 } & \multicolumn{1}{c}{ Two Process } & \multicolumn{1}{c}{ Shared Outcome } \\
\hline S1 trials: $\mathrm{S} 1-\mathrm{R} 1-\mathrm{O} 1 / \mathrm{O} 2$ & $\mathrm{~S} 1 \rightarrow$ expect $\mathrm{O} 1$ & $\mathrm{~S} 1 \rightarrow$ expect $\mathrm{O} 1$ \\
& expect $\mathrm{O} 1 \rightarrow \mathrm{R} 1$ & $\mathrm{R} 1 / \mathrm{R} 2 \leftarrow$ expect $\mathrm{O} 1$ \\
$\mathrm{~S} 2$ trials: $\mathrm{S} 2-\mathrm{R} 2-\mathrm{O} 1 / \mathrm{O} 2$ & $\mathrm{~S} 2 \rightarrow \operatorname{expect~} \mathrm{O} 2$ & $\mathrm{~S} 2 \rightarrow$ expect O2 \\
& expect O2 $\rightarrow \mathrm{R} 2$ & $\mathrm{R} 1 / \mathrm{R} 2 \leftarrow$ expect $\mathrm{O} 2$ \\
\hline
\end{tabular}

Note-S, initial stimulus; $R$, comparison response reinforced; $O$, outcome used to reinforce a correct response. relative facilitative effect of differential outcomes in acquisition. In other words, differential-sample responding cues alone appeared to facilitate acquisition about as well as differential-sample responding and differential-outcome expectancies combined. But differential-outcome expectancies did facilitate delay performance: The differentialoutcomes group (i.e., pigeons provided with both differential-sample responding cues and differential-outcome expectancies) performed substantially better on long-delay tests than did the control group (i.e., pigeons provided with differential-sample responding cues, but not differentialoutcome expectancies). Thus, in the present experiment, it was expected that the effects of differential outcomes (beyond those of differential-sample responding) would be most evident in delay performance.

Second, when we have used differential outcomes of food and no food, we have found biases in the form of a strong preference for the comparison associated with the food outcome (e.g., Zentall \& Sherburne, 1994). Such a bias can mask group differences in acquisition rate and can affect relative delay performance as well. In the present experiment, one would expect such a bias to affect the SCO group (in which comparison-outcome associations were differential), but not the SO and NDO groups (in which comparison-outcome associations were nondifferential).

Thus, in the present experiment, the need to control for differential-sample responding and differential outcomes associated with comparison responses prevents distinguishing between the two theories on the basis of differences in performance at each delay. Instead, the critical focus of Experiment 1 was on the relative decline in performance as a function of delay. If differential response-generated expectancies (i.e., backward associations) contribute to the DOE, then SCO group performance should be less affected by delay than SO group performance. If, on the other hand, the use of differential sample-generated expectancy cues is sufficient to account for the DOE, then SCO and SO group performances should be similarly affected by delay, and both groups should show a DOE (i.e., SCO and SO group performances should be less affected by delay than is NDO group performance).

\section{EXPERIMENT 1}

\section{Method}

\section{Subjects}

Subjects were 12 White Carneaux pigeons purchased from the Palmetto Pigeon plant (Sumter, SC). All pigeons had served in a previous experiment involving simple hue discriminations. Purina Pro Grains, mostly provided during experimental sessions, maintained the pigeons at $80 \%-85 \%$ of their free-feeding weights. The birds were housed in individual wire-mesh cages with water and grit freely available. The colony was maintained under a $12: 12-\mathrm{h}$ light:dark cycle.

\section{Apparatus}

Experimental sessions were conducted in a BRS/LVE (Beltsville, MD) operant chamber. The chamber measured $37.5 \mathrm{~cm}$ high, 28.0 $\mathrm{cm}$ from front panel to back wall, and $35 \mathrm{~cm}$ across the front panel. 
Stimuli were projected on the center of three of five horizontally aligned rectangular $(3.5 \times 3.0 \mathrm{~cm})$ response keys $(1.0 \mathrm{~cm}$ between edges of adjacent keys) by means of in-line stimulus projectors (Industrial Electronics Engineering, Series 10, Van Nuys, CA) mounted behind each key. The center key could be illuminated with a red hue (Kodak Wratten filter no. 26), a green hue (Kodak Wratten filter no. 60 ), a white circle (outside diameter of $16 \mathrm{~mm}$, inside diameter of 13 $\mathrm{mm})$, or a white dot (5-mm diameter). Three white vertical lines (each $13 \mathrm{~mm}$ long $\times 3 \mathrm{~mm}$ wide and separated by $3 \mathrm{~mm}$ ) or three white horizontal lines (same dimensions as the vertical lines), presented on a black background, could be projected onto each of the side keys. The feeder, which contained mixed grain, was mounted behind the front panel so that, when raised, it was lit and accessible through a $5.1 \times 5.7 \mathrm{~cm}$ aperture centered horizontally on the panel. The bottom edge of the aperture was $7 \mathrm{~cm}$ above the wire-mesh floor. A shielded houselight (GE 1820 lamp) was mounted $4 \mathrm{~cm}$ above the response keys. Extraneous sounds were masked by white noise at 72 $\mathrm{dB}$ presented through a speaker located on the left front wall of the chamber and by the sound of an exhaust fan mounted on the exterior of the chamber. Experimental contingencies and data recording were controlled by a microcomputer located in an adjacent room.

\section{Procedure}

Because of their previous experience, the pigeons were accustomed to the operant chamber and to pecking response keys. Therefore, training on the conditional discrimination began immediately.

Zero-sec delay training. Each trial began with presentation of either a red or a green stimulus on the center key. All pigeons were required to respond 10 times (Behavior 1 [B1], FR 10) to one of the initial stimuli (S1) and to withhold responding (B2, Behavior 2; DRO $6 \mathrm{sec}$ ) to the other initial stimulus (S2). For 2 of the pigeons in each of the three groups, S1 was red and S2 was green. For the other pigeons, S1 was green and S2 was red.

On 96 of the trials in each session, offset of the initial stimulus ( $\mathrm{S} 1$ or $\mathrm{S} 2$ ) was followed immediately either by a 1.5 -sec presentation of mixed grain accompanied by the feeder light (food outcome, O1) or by a 1.5-sec presentation of the feeder light alone (no-food outcome, O2). These simple discrimination trials are designated as $\mathrm{S} 1$ simple and $\mathrm{S} 2$ simple trials. The relative proportions of $\mathrm{Ol}$ and $\mathrm{O} 2$ on $\mathrm{S} 1$ and $\mathrm{S} 2$ simple trials varied over the three groups. The 10 sec houselight-illuminated intertrial interval (ITI) followed the outcome on each trial.

On the remaining 48 trials in each session, $\mathrm{S} 1$ and $\mathrm{S} 2$ served as samples in a conditional discrimination (designated as S1 and S2 conditional trials). On these trials, offset of the sample resulted in presentation of vertical- and horizontal-line comparisons on the side keys. On $\mathrm{S} 1$ conditional trials, a single peck to the vertical lines (R1) was reinforced with either $\mathrm{O} 1$ or $\mathrm{O} 2$. On S2 conditional trials, a single peck to the horizontal lines (R2) was reinforced with either $\mathrm{O} 1$ or $\mathrm{O} 2$. The relative proportions of $\mathrm{O} 1$ and $\mathrm{O} 2$ that followed each of the correct-comparison responses varied over the three groups. Again, the ITI followed the outcome on each trial. Incorrect responses (i.e., those to the vertical lines on S2 conditional trials and those to the horizontal lines on $\mathrm{S} 1$ conditional trials) were followed by the ITI and repeats (up to five) of the trial until a correctcomparison response was made. The color of the initial stimulus, position of the correct comparison, and type of discrimination (simple or conditional) required on a trial were balanced within blocks of 12 trials. Trial order within these blocks was random except for the restriction that no more than three consecutive trials involved the same correct-comparison position, hue stimulus, or type of discrimination. Two sequences of trial order were double-alternated over sessions.

SO group. On each $\mathrm{S} 1$ simple trial, $\mathrm{O} 1$ occurred and on each $\mathrm{S} 2$ simple trial, $\mathrm{O} 2$ occurred $[p(\mathrm{O} 1 \mid \mathrm{S} 1$ simple $)=1.0, p(\mathrm{O} 2 \mid \mathrm{S} 1$ simple $)=0, p(\mathrm{O} 1 \mid \mathrm{S} 2$ simple $)=0$, and $p(\mathrm{O} 2 \mid \mathrm{S} 2$ simple $)=1.0]$. On $\mathrm{S} 1$ and $\mathrm{S} 2$ conditional trials, however, the outcomes were nondifferen- tial $[p(\mathrm{O} 1 \mid \mathrm{S} 1$ conditional| $\mathrm{R} 1)=p(\mathrm{O} 2 \mid \mathrm{S} 1$ conditional $\mid \mathrm{R} 1)=$ $p(\mathrm{O} 1 \mid \mathrm{S} 2$ conditional $\mid \mathrm{R} 2)=p(\mathrm{O} 2 \mid \mathrm{S} 2$ conditional $\mid \mathrm{R} 2)=.50]$.

SCO group. On a random five sixths of the $S 1$ simple trials and a random one sixth of the $\mathrm{S} 2$ simple trials, $\mathrm{O} 1$ outcomes occurred. On the other sixth of the Sl simple trials and the other five sixths of the $\mathrm{S} 2$ simple trials, $\mathrm{O} 2$ outcomes occurred $[p(\mathrm{O} 1 \mid \mathrm{S} 1$ simple $)=$ $.83, p(\mathrm{O} 2 \mid \mathrm{S} 1$ simple $)=.17, p(\mathrm{O} 1 \mid \mathrm{S} 2$ simple $)=.17$, and $p(\mathrm{O} 2 \mid \mathrm{S} 2$ simple $)=.83$ ]. The same contingencies were in effect on conditional discrimination trials involving a correct-comparison response $[p(\mathrm{O} 1 \mid \mathrm{S} 1$ conditional $\mid \mathrm{R} 1)=.83, p(\mathrm{O} 2 \mid \mathrm{S} 1$ conditional $\mid \mathrm{R} 1)=.17$, $p(\mathrm{O} 1 \mid \mathrm{S} 2$ conditional $\mathrm{R} 2)=.17$, and $p(\mathrm{O} 2 \mid \mathrm{S} 2$ conditional $\mid \mathrm{R} 2)=$ .83]. Thus, for the SCO pigeons, the differential-outcome probabilities associated with the "samples" were the same as those in the $\mathrm{SO}$ group [given correct-comparison responding, $p(\mathrm{O} 1)=.83$ for $\mathrm{S} 1$ and $p(\mathrm{O} 1)=.17$ for $\mathrm{S} 2]$.

NDO group. $\mathrm{O} 1$ and $\mathrm{O} 2$ outcomes occurred equally often on $\mathrm{S} 1$ simple and $\mathrm{S} 2$ simple trials $[p(\mathrm{O} 1 \mid \mathrm{S} 1$ simple $)=p(\mathrm{O} 2 \mid \mathrm{S} 1$ simple $)=$ $p(\mathrm{O} 1 \mid \mathrm{S} 2$ simple $)=p(\mathrm{O} 2 \mid \mathrm{S} 2$ simple $)=.50]$. The same contingencies were in effect on conditional trials involving a correctcomparison response $[p(\mathrm{O} 1 \mid \mathrm{S} 1$ conditional $\mid \mathrm{R} 1)=p(\mathrm{O} 2 \mid \mathrm{S} 1$ conditional $\mid \mathrm{R} 1)=p(\mathrm{O} 1 \mid \mathrm{S} 2$ conditional $\mid \mathrm{R} 2)=p(\mathrm{O} 2 \mid \mathrm{S} 2$ conditional $\mid \mathrm{R} 2)=$ $.50]$. Thus, for the NDO pigeons, the outcome probabilities associated with correct-comparison responding were the same as those for the SO group.

Testing with mixed delays. After each subject reached a criterion of at least $90 \%$ correct performance for two consecutive sessions, it began delay testing. As in training, a session consisted of 144 trials ( 96 simple trials and 48 conditional trials). On the conditional trials, mixed delays $(0,1,2$, and $4 \mathrm{sec})$ were introduced between the initial stimuli and the comparisons. On simple trials, the delays were introduced between the initial stimuli and the trial outcomes. Each of the delays occurred equally often and was counterbalanced over trial type. All stimuli, including the houselight, were off during the delays. When a pigeon had completed six sessions of testing with a given delay set, the delays were doubled and the bird was tested for another six sessions. Delay testing continued until the birds had completed six sessions at each of the following sets of mixed delays: $0,1,2$, and $4 \mathrm{sec}$ (Delay Set 1); 0,2, 4, and $8 \mathrm{sec}$ (Delay Set 2); 0, 4, 8, and $16 \mathrm{sec}$ (Delay Set 3); and $0,8,16$, and $32 \mathrm{sec}$ (Delay Set 4). A 05 level of significance was adopted for all analyses.

\section{Results}

As expected, there was no evidence of a typical DOE in acquisition. Although an analysis of variance (ANOVA) performed on the sessions-to-criterion data revealed a significant effect of group $[F(2,9)=30.06]$, posthoc analyses (Tukey-Kramer HSD test) showed that both Group SO and Group NDO acquired the task significantly faster $(M \mathrm{~s}=10.5$ and 14.5 sessions, respectively) than did Group SCO $(M=28.5$ sessions $), q=11.19$ and 7.19 for SO-versus-SCO and NDO-versus-SCO group comparisons, respectively. The difference in acquisition rates between Groups SO and NDO, however, was not significant, $q=-2.81$.

Effects found during testing with the first three delay tests were modest. But all significant effects found during testing with these shorter delays were also present during testing with the longest delay set. Thus, data analysis focused on Delay Set 4. A three-way mixed ANOVA (with group as a between-subjects factor and delay and sample type as within-subjects factors) was performed on these data. 
An overall tendency for performance to be higher on S1 than on S2 trials was reflected in a significant sample type effect $[F(1,9)=19.35 ; M=74.9 \%$ correct and $65.6 \%$ correct for S1 and S2 trials, respectively]. And a tendency for $\mathrm{S} 1$ trial performance to be more affected by delay than S2 trial performance was reflected in a significant sample type $\times$ delay interaction $[F(3,27)=$ 14.57]. The SCO group comparison bias was manifested in a significant sample type $\times$ group interaction $[F(2,9)$ $=15.51$; mean SCO group performance on S1 sample trials vs. S2 sample trials $=81.8 \%$ correct and $56.4 \%$ correct, respectively; mean SO group performance on S1 sample trials vs. S2 sample trials $=78.6 \%$ correct and $80.9 \%$ correct, respectively; mean NDO group performance on S1 sample trials vs. S2 sample trials $=64.2 \%$ correct and 59.5\% correct, respectively]. But this relative bias in the SCO group was not affected significantly by delay $[F(6,27)=1.09]$ for the three-way interaction.

The effect of group $[F(2,9)=31.25]$ was significant, however, and was accompanied by a significant group $X$ delay interaction $[F(6,27)=3.11]$. To determine whether or not the interaction reflected a DOE, we performed a second three-way ANOVA, in which the group factor consisted of differential outcomes (SO group and SCO group data combined) versus nondifferential outcomes (NDO group data). This analysis revealed significant effects of group $[F(1,9)=40.50]$ and of the group $\times$ delay interaction $[F(3,27)=5.11]$. As can be seen in Figure 1, differential sample-outcome associations not only facilitated overall performance, but also provided resistance to the effect of delay on performance. In addition, planned

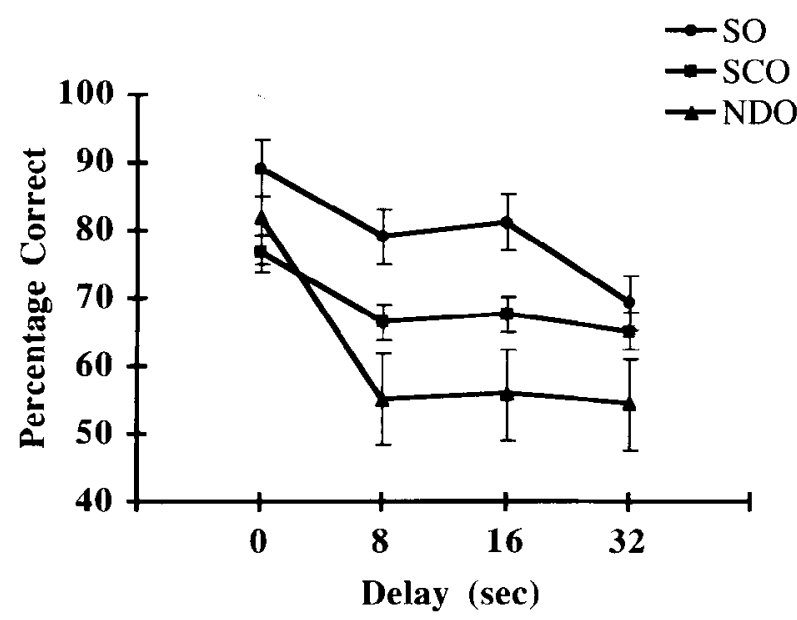

Figure 1. Experiment 1: Performance of the three groups over six sessions of testing with Delay Set 4 . Vertical lines represent standard errors of the means. For the sample-outcome (SO) group, samples were associated with differential outcomes but comparisons were not. For the sample-comparison-outcome (SCO) group, both samples and comparisons were associated with differential outcomes. For the nondifferential-outcome (NDO) group, neither samples nor comparisons were associated with differential outcomes. comparisons revealed that the SO group performed at a significantly higher level, overall, than did the SCO group $[F(1,9)=22.01]$. This difference, however, did not interact significantly with delay $[F(3,27)=1.12]$. Thus, differential response-outcome associations were shown to have an adverse effect on the facilitation of overall performance provided by differential sample-outcome associations. The delay-mediation benefit provided by differential sample-outcome associations, however, did not appear to be affected by whether or not the responseoutcome associations were differential.

\section{Discussion}

The performance of the two groups that had differential sample-outcome associations ( $\mathrm{SCO}$ and $\mathrm{SO}$ ) was significantly better overall and was significantly more resistant to the effect of delay than that of the group that had nondifferential sample-outcome associations (NDO).

There was no indication, however, that differential response-outcome associations provided any facilitation beyond that contributed by differential sample-outcome associations. In fact, the SCO group performed significantly worse, overall, than did the SO group during testing with the longest delay set. This deficit apparently resulted from the SCO group response bias, a preference attributable to differential response-outcome associations. But the absence of a difference between Groups SCO and SO in rates of "forgetting" suggests that no underlying facilitative effect of differential response-outcome associations was being obscured by the bias. This conclusion is further strengthened by the finding that the relative comparison bias did not increase with delay.

\section{EXPERIMENT 2}

The results of Experiment 1 demonstrated that at least one manifestation of the DOE, superior resistance to the effect of delay on performance, can be obtained in the absence of differential response-outcome associations. These results implicate differential sample-generated expectancies as the sole source of the facilitation provided by differential outcomes.

Experiment 2 was designed to confirm and extend these findings using a transfer procedure. The primary question of interest was whether or not differential expectancies established independently of the differentialoutcomes conditional discrimination could effectively cue comparison responses.

After the pigeons from Experiment 1 were retrained on the original 0 -sec-delay conditional discrimination involving hue samples and line comparisons, they were trained to associate two new initial stimuli (i.e., circle and dot shapes) with differential outcomes (i.e., a high probability of $\mathrm{O} 1 \mathrm{vs.}$ a high probability of $\mathrm{O} 2$ ) in a simple successive discrimination. In order to preclude positive transfer based on the use of differential sample-responding cues, DRO (B2) schedules were required to both of the 
Table 4

Design of Experiment 2

\begin{tabular}{|c|c|c|c|}
\hline Group & Conditional Training & Simple Training & Transfer Test \\
\hline \multirow[t]{2}{*}{ SO } & $\mathrm{S} 1-\mathrm{B} 1-(\operatorname{expect} \mathrm{O} 1)-\mathrm{R} 1-$ & $\mathrm{S} 3-\mathrm{B} 2($ expect $\mathrm{O} 1)$ & $\mathrm{R} 1$ \\
\hline & $\mathrm{S} 2-\mathrm{B} 2-(\exp$ & $-($ expect 02$)$ & B2-R2 \\
\hline \multirow[t]{2}{*}{$\mathrm{SCO}$} & $1-(\operatorname{expect} \mathrm{O} 1)$ & (expect $\mathrm{O} 1)$ & S3-B2-R1 \\
\hline & S2-1 & o2) & $2-\mathrm{R} 2$ \\
\hline \multirow[t]{2}{*}{ NDO } & $\mathrm{S} 1-\mathrm{B} 1-(\operatorname{expect} \mathrm{O} 1 / \mathrm{O} 2)-\mathrm{R} 1-(\operatorname{expect} \mathrm{O} 1 / \mathrm{O} 2)$ & S3-B2(expect O1) & $\mathrm{S} 3-\mathrm{B} 2-\mathrm{R} 1$ \\
\hline & $\mathrm{S} 2-\mathrm{B} 2-(\operatorname{expect} \mathrm{O} 1 / \mathrm{O} 2)-\mathrm{R} 2-(\operatorname{expect} \mathrm{O} 1 / \mathrm{O} 2)$ & $\mathrm{S} 4-\mathrm{B} 2-(\operatorname{expect} \mathrm{O} 2)$ & $\mathrm{S} 4-\mathrm{B} 2-\mathrm{R} 2$ \\
\hline
\end{tabular}

Note-S1, Sample 1 (red hue for half the birds, green hue for the others); S2, Sample 2 (green hue for half the birds, red for the others); S3, Stimulus 3 (circle shape); S4, Stimulus 4 (dot shape); B1, Behavior 1 (fixed ratio 10); B2, Behavior 2 (differential reinforcement of other behavior $6 \mathrm{sec}$ ); Expect, expectancy presumed to have been established; O1, Outcome 1 (food); O2, Outcome 2 (no food, feeder light); R1, correct comparison Response 1 (choice of a vertical line); R2, correct comparison Response 2 (choice of a horizontal line). The test trials were conducted with mixed delays $(0,1,2$, and $4 \mathrm{sec})$ inserted between $S$ presentations and outcomes (simple trials) and between $S$ presentations and comparison presentations (conditional trials).

shape stimuli. During test, the hue samples were replaced with the shapes from the simple successive discrimination.

It was expected that if the $\mathrm{SO}$ and $\mathrm{SCO}$ groups were, indeed, using differential-outcome expectancies as cues for choice in the conditional discrimination, they should perform significantly better on the transfer task than the NDO group. In addition, it was expected that any facilitation provided by the sharing of sample- and responseoutcome associations should be reflected in superior performance by the SCO group relative to that of the SO group. Finally, it was expected that the extent to which the pigeons' choices in training had been controlled by sample responding cues (i.e., B1, pecking, vs. B2, not pecking) would be reflected in a bias, in test, to respond to the B2-associated comparison (R2). The design of Experiment 2 is presented in Table 4 .

\section{Method}

\section{Subjects}

The subjects were those from Experiment 1.

\section{Apparatus}

The pigeons were tested in the chamber that was used in Experiment 1 .

\section{Procedure}

When a subject's delay testing in Experiment 1 was completed, it was retrained on the 0 -sec delay task until the criterion performance level was regained. The pigeon was then exposed to 144 trial sessions of simple discrimination training with new stimuli. A trial began with presentation of either a circle or dot shape on the center key. Regardless of which shape was presented, the birds were required to not respond to the shape stimulus in order to obtain the trial outcome. When no pecks had occurred to the stimulus for $6 \mathrm{sec}$ (B2, DRO 6 sec schedule), either $\mathrm{O} 1$ or $\mathrm{O} 2$ occurred.

The circle shape (S3) was followed by $\mathrm{O} 1$ on five sixths of the trials and by $\mathrm{O} 2$ on one sixth of the trials. The dot shape (S4) was followed by $\mathrm{O} 2$ on five sixths of the trials and by $\mathrm{O} 1$ on one sixth of the trials. Thus, for birds in both the SO and SCO groups, the $p(\mathrm{O} 1)$ and the $p(\mathrm{O} 2)$ associated with $\mathrm{S} 3$ were the same as that for $\mathrm{S} 1$ (given choice of the correct comparison), and the $p(\mathrm{Ol})$ and the $p(\mathrm{O} 2)$ associated with $\mathrm{S} 4$ were the same as that for $\mathrm{S} 2$. For birds in the NDO group, however, the differential-outcome probabilities associated with S3 and S4 were not shared by S1 and S2.
Each pigeon was required to meet a stimulus-responding criterion on the shape stimulus task: for a single session, the number of responses to each of the shapes (S3 and S4) was required to be $\leq 36$ (an average of $<0.5$ responses per trial to each stimulus). If a bird failed to reach the stimulus-responding criterion within 15 sessions (as did 6 of the 10 birds), it was placed on a correction procedure that involved "punishment" of any response to a shape stimulus by removal of the stimulus and omission of the trial outcome. One bird (in the SO group) failed to reach the criterion after 50 sessions under these conditions and was dropped from the study at this point. A 2nd bird (NDO group) was transferred prior to achieving the criterion (experimenter error) and was also dropped from the study.

When a pigeon reached the stimulus-responding criterion on the shape stimulus task, it was retrained during the next session with the Phase 1 task. Phase 1 sessions and shape stimulus sessions were alternated until the correct performance and stimulus responding criteria were regained for two consecutive sessions (i.e., a singlecriterion session on each task). The pigeon was tested on the following session by replacing S1 with S3 and replacing S2 with S4 on all trials (both simple and conditional) of the training task. Responses reinforced during test were $\mathrm{R} 1$ after $\mathrm{S} 3$ presentations and $\mathrm{R} 2$ after $\mathrm{S} 4$ presentations.

Disruption of any previously established sample-outcome and comparison-outcome associations was minimized during testing by using an SO procedure for birds in the NDO and SO groups and an SCO procedure for birds in the SCO group. That is, for NDO and $\mathrm{SO}$ birds, all $\mathrm{S} 1$ simple trials ended with $\mathrm{O} 1$, all $\mathrm{S} 2$ simple trials ended with $\mathrm{O} 2$, and correct responses on conditional trials were nondifferentially reinforced. For SCO birds, both S1 simple and S1 conditional correct-response trials ended with $\mathrm{Ol}$ on five sixths of the trials and both $\mathrm{S} 2$ simple and $\mathrm{S} 2$ conditional correct-response trials ended with $\mathrm{O} 2$ on five sixths of the trials. Thus, given correct responding, the outcome associations established in previous phases were maintained in all groups [i.e., $p(\mathrm{O} 1 / \mathrm{S} 3)$ and $p(\mathrm{O} 2 / \mathrm{S} 4)$ $=.83$ for all groups; $p(\mathrm{O} 1 / \mathrm{R} 1)$ and $p(\mathrm{O} 2 / \mathrm{R} 2)=.83$ for SCO birds; $p(\mathrm{O} 1 / \mathrm{R} 1)$ and $p(\mathrm{O} 2 / \mathrm{R} 2)=.50$ for $\mathrm{NDO}$ and $\mathrm{SO}$ birds]. Testing was with mixed delays $(0,1,2$, and $4 \mathrm{sec}$, balanced over trial type $)$ and testing continued for 10144 -trial sessions.

\section{Results}

A three-way ANOVA with group (SO vs. SCO vs. NDO), trial type (S3 vs. S4), and delay $(0,1,2$, and $4 \mathrm{sec}$ ) as factors, was performed on the data from the first samplereplacement test session. The result of most interest from the transfer test was a significant effect of group $[F(2,7)=$ 


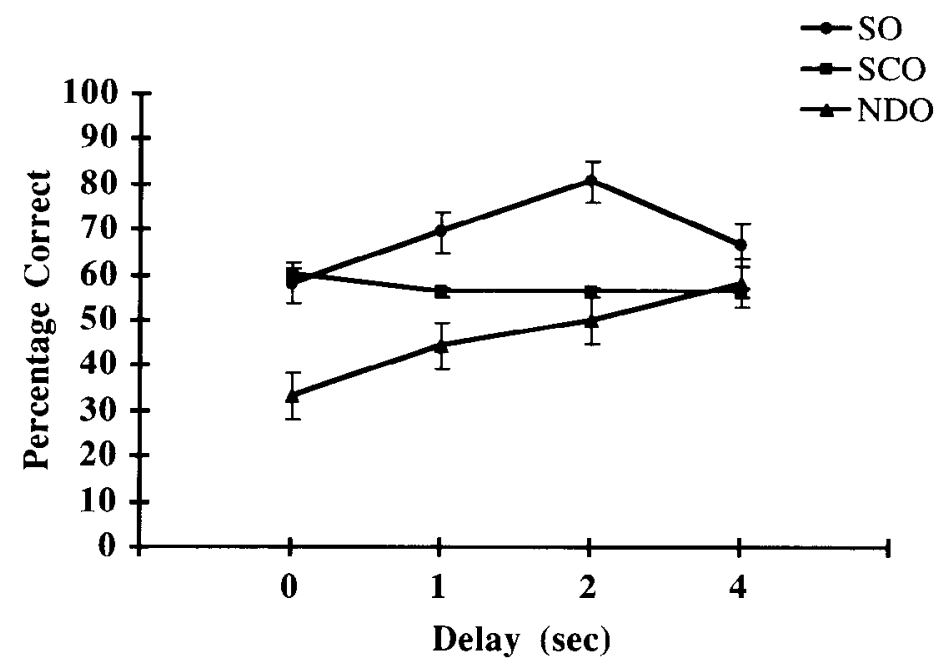

Figure 2. Experiment 2: Performance on the single-session sample-replacement test. Vertical lines represent standard errors of the means. Shapes, differentially associated with the two outcomes, and to which the pigeons were required to withhold responding (B2), were substituted for the hue samples (to which differential responding was required) used in training. Responses counted as correct were those consistent with use of outcome expectancies as choice cues in the two differential-outcomes groups. For the sample-outcome (SO) group, samples were associated with differential outcomes but comparisons were not. For the sample-comparison-outcome (SCO) group, both samples and comparisons were associated with differential outcomes. For the nondifferentialoutcome (NDO) group, neither samples nor comparisons were associated with differential outcomes.

6.52; see Figure 2]. Planned comparisons were conducted to determine whether or not the effect reflected a DOE (i.e., superior DO performance relative to NDO performance), and to determine whether or not the DO groups (SO vs. SCO) differed from each other. Although overall DO (SO and SCO groups combined) performance was significantly better than NDO performance $[F(1,7)$ $=9.08]$, there was no significant difference in overall performance between the two DO groups $[F(1,7)=3.96]$. Thus, the consistency of outcome associations between the hue samples and shape "samples" facilitated performance, but there was no evidence of an additional effect of differential response-outcome associations on overall performance.

The effect of trial type was also significant in the overall analysis $[F(1,7)=15.59]$. As can be seen in Figure 3, the comparison that had been associated with S2 was chosen correctly more often than was the comparison that had been associated with S1. This strong response bias is not surprising. Recall that differential responding to the samples (FR to S1, DRO to S2) was reinforced for all pigeons, but nondifferential responding (DRO) was required to both S3 and S4. Thus, to a considerable extent, sample responding (or its absence) controlled comparison responses on test trials.

The results presented in Figure 3 also suggest that this trial-type performance difference varied as function of delay in the SO group (left panel), but that the difference was relatively unaffected by delay in the other two groups (center and right panels). A marginally significant three-way interaction in the overall analysis $[F(6,21)=$ 2.53 ], as well as significant trial type $\times$ delay and threeway interactions in the SO-versus-SCO analysis $[F \mathrm{~s}(3,21)$ $=3.45$ and 3.96 , respectively] confirmed this apparent difference. SO group performance tended to be improved by delaying comparison presentation on trials initiated by the O1-associated shape. On 2-sec-delay test trials, this improvement was, in fact, sufficient to eliminate the trial-type performance difference otherwise observed.

Although overall matching accuracy for the NDO group appears to be below chance at the 0-sec delay, the magnitude of the bias to choose the comparison associated in training with the absence of responding to the sample was highly variable, and this level of matching accuracy was not statistically below chance $[F(1,2)=3.00]$.

\section{Discussion}

The transfer test results confirmed the use of expectancies based on differential sample-outcome associations as choice cues. The performance of the SO and SCO groups was significantly superior to that of the NDO group when the samples were replaced by new stimuli associated with the same differential-outcome probabilities. Transfer performance was not further facilitated, however, by differential comparison-outcome associations; SCO group transfer performance was not superior to that of the SO group. These results are consistent with those of Experiment 1. 


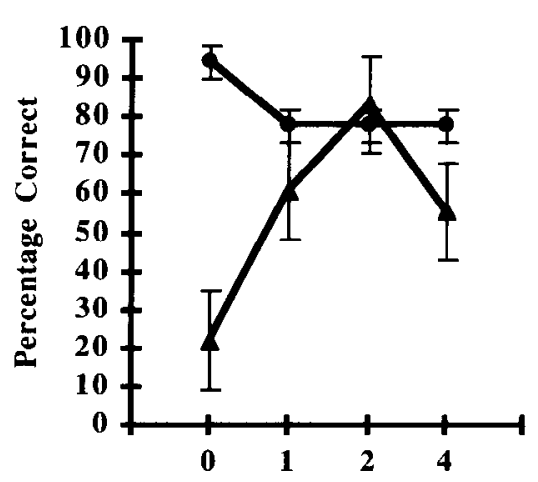

Sample Outcomes

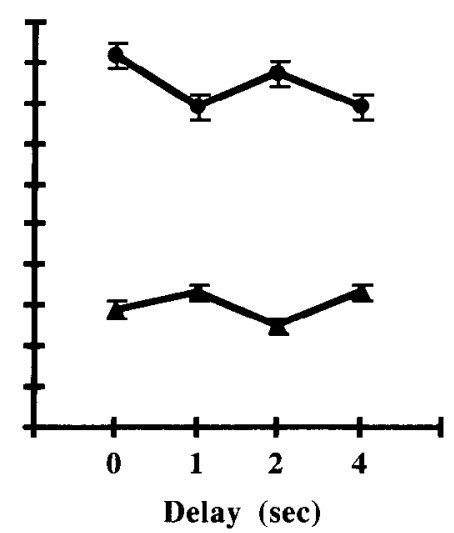

Sample Comparison Outcomes

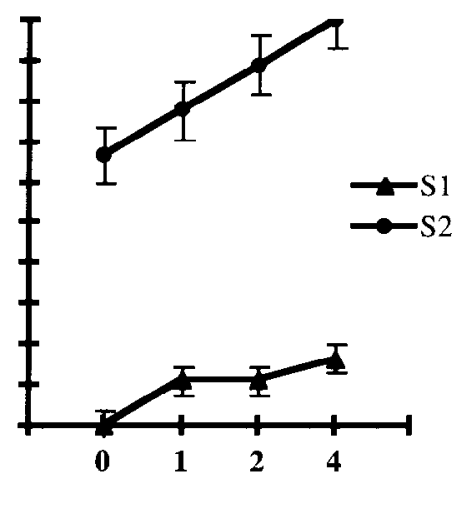

Nondifferential Outcomes

Figure 3. Experiment 2: Performance on the sample-replacement test as a function of whether the sample replaced was $\mathbf{S 1}$ (B1) or $\mathbf{S 2}$ (B2). Vertical lines represent standard errors of the means. For the sample-outcome group (left panel), samples were associated with differential outcomes, but comparisons were not. For the sample-comparison-outcome group (middle panel), both samples and comparisons were associated with differential outcomes. For the nondifferential-outcome group (right panel), neither samples nor comparisons were associated with differential outcomes.

In addition, differential-sample responding was shown to exert considerable control over comparison responses. Although this effect was not surprising in the NDO group, the degree of control exerted by sample responding in the SCO group was not expected, given the large differentialoutcomes transfer effects we obtained in a previous experiment in which differential sample-responding cues were eliminated (Sherburne \& Zentall, 1995). One possible explanation for this discrepancy is that the differentialoutcome probabilities used in the present study $(83 \%$ vs. $17 \%$ ) simply do not provide expectancy cues as effective as those produced by the nonprobabilistic differential outcomes used in the earlier study ( $100 \%$ vs. $0 \%)$. Thus, differential-sample responding cues may exert more control over responding in a probabilistic differential-outcomes task than in a nonprobabilistic differential-outcomes task.

A second possible reason for this difference in transfer effects may be that the pigeons in the present experiment were never explicitly instructed that sample responding cues should not be used as the basis for choice, whereas pigeons in the comparable groups of our earlier study were trained not to use such cues. Thus, it may be that pigeons normally use differential sample responding cues, in addition to differential outcome-expectancy cues, in a differential-outcomes task.

The test performance of the SO group was in some respects quite similar to that of the SCO group. Although the DOE obtained (i.e., overall DO performance was significantly better than that of the NDO group) indicates some control by differential expectancies, differential sample responding also exerted substantial control over choice.

More interesting is the change in SO group performance observed across delays. Paradoxically, choice be- came more accurate as a result of delaying presentation of the comparisons. This increase in SO performance at intermediate delays suggests that as the time between sample offset and comparison presentation passed, expectancy cues gained increasing control over responding. At the 2-sec delay, SO group performance was quite high ( $80 \%$ correct). SO group performance did appear to drop as the delay was increased beyond $2 \mathrm{sec}$, however. This drop suggests that as the delay was extended, some forgetting of the outcome expectancy took place.

It has been argued that differential-outcome expectancies, because of their salience, come to overshadow other available cues in a differential-outcomes task (see Urcuioli, 1984, 1985, 1990). The SO group transfer performance suggests, instead, that outcome expectancies enhance performance by providing choice cues in addition to those available in a similar task trained with nondifferential outcomes (Peterson, 1984), and that control by the different types of cues may vary with the retention interval.

\section{GENERAL DISCUSSION}

The results of these experiments provide support for the two-process account of the DOE. The differentialoutcome expectancies produced by differential sampleoutcome associations appear to be sufficient to account for the facilitated performance observed in delayed conditional discriminations with pigeons. In fact, when these differential outcomes have different hedonic value (as they often do) and differential-outcome expectancies do not also result in differential response-outcome associations, a substantially stronger DOE may be produced, because comparison-response biases are less likely to occur. Thus, under the present conditions, providing differential 
response-outcome associations does not appear to contribute to the DOE. Furthermore, the finding that sampleoutcome associations, in the absence of comparisonoutcome associations, can produce substantial facilitation of matching performance suggests the importance of these associations in the typical DOE. In addition, the results of the present experiments suggest that both differentialsample responding and differential sample-generated outcome expectancies are encoded in a differential-outcomes conditional discrimination, and that with short delays, the differential sample behavior may strongly influence comparison responses. The results of the present experiments suggest that it is not until the sample-comparison delay becomes relatively long that outcome expectancies gain substantial control of choice behavior.

\section{REFERENCES}

Carlson, J. G., \& Wielkiewicz, R. M. (1972). Delay of reinforcement in instrumental discrimination learning of rats. Journal of Comparative \& Physiological Psychology, 81, 365-370.

CARLSON, J. G., \& WielKIEWICZ, R. M. (1976). Mediators of the effects of magnitude of reinforcement. Learning \& Motivation, 7, 184-196.

Colwill, R. M., \& Rescorla, R. A. (1985). Postconditioning devaluation of a reinforcer affects instrumental responding. Journal of $E x-$ perimental Psychology: Animal Behavior Processes, 11, 120-132.

DElong, R. E., \& Wasserman, E. A. (1981). Effects of differential reinforcement expectancies on successive matching-to-sample performance in pigeons. Journal of Experimental Psychology: Animal Behavior Processes, 7, 394-412.

DeMarse, T., \& Urcuioli, P. J. (1993). Enhancement of matching acquisition by differential comparison-outcome associations. Journal of Experimental Psychology: Animal Behavior Processes, 19, $317-$ 326.

FedorChaK, P. M., \& Bolles, R. C. (1986). Differential outcome effect using a biologically neutral outcome difference. Journal of Experimental Psychology: Animal Behavior Processes, 12, 125-130.

JENKINS, H. M., \& MoORE, B. R. (1973). The form of the auto-shaped response with food or water reinforcers. Journal of the Experimental Analysis of Behavior, 20, 163-181.

OVERMier, J. B., \& LAWry, J. A. (1979). Pavlovian conditioning and the mediation of behavior. In G. H. Bower (Ed.), The psychology of learning and motivation (Vol. 13, pp. 1-55). New York: Academic Press.

Peterson, G. B. (1984). How expectancies guide behavior. In H. L. Roitblat, T. G. Bever, \& H. S. Terrace (Eds.), Animal cognition (pp. 135148). Hillsdale, NJ: Erlbaum.
Peterson, G. B., Wheeler, R. L., \& Trapold, M. A. (1980). Enhancement of pigeons' conditional discrimination performance by expectancies of reinforcement and nonreinforcement. Animal Learning \& Behavior, 8, 22-30.

RESCORLA, R. A. (1992). Response outcome versus outcome-response associations in instrumental learning. Animal Learning \& Behavior, 20, 223-232.

Sherburne, L. M., \& Zentall, T. R. (1995). Pigeons transfer between conditional discriminations with differential outcomes in the absence of differential-sample-responding cues. Animal Learning \& Behavior, 23, 273-279.

Trapold, M. A. (1970). Are expectancies based on different reinforcing events discriminably different? Learning \& Motivation, 1, 129140 .

TraPold, M. A., \& OVermier, J. B. (1972). The second learning process in instrumental learning. In A. H. Black \& W. F. Prokasy (Eds.), Classical conditioning II: Current research and theory (pp. 427452). New York: Appleton-Century-Crofts.

URCUIOLI, P. J. (1984). Overshadowing in matching-to-sample: Reduction in sample-stimulus control by differential sample behaviors. Animal Learning \& Behavior, 12, 256-264.

URCUIOLI, P. J. (1985). On the role of differential sample behaviors in matching-to-sample. Journal of Experimental Psychology: Animal Behavior Processes, 11, 502-519.

URCUIOLI, P. J. (1990). Some relationships between outcome expectancies and sample stimuli in pigeons' delayed matching. Animal Learning \& Behavior, 18, 302-314.

Urcuioli, P. J., \& DeMarse, T. (1994). On the relationship between differential outcomes and differential sample responding in matchingto-sample. Journal of Experimental Psychology: Animal Behavior Processes, 20, 249-263.

Urcuioli, P. J., \& DeMarse, T. (1996). Associative processes in differential outcome discriminations. Journal of Experimental Psychology: Animal Behavior Processes, 22, 192-204.

URCUioli, P. J., \& HoNiG, W. K. (1980). Control of choice in conditional discriminations by sample-specific behaviors. Journal of $E x$ perimental Psychology: Animal Behavior Processes, 6, 251-277.

Zentall, T. R., Hogan, D. E., Howard, M. M., \& Moore, B. S. (1978). Delayed matching in the pigeon: Effect on performance of sample-specific observing responses and differential delay behavior. Learning \& Motivation, 9, 202-218.

Zentall, T. R., \& Sherburne, L. M. (1994). The role of differential sample responding in the differential outcomes effect involving delayed matching by pigeons. Journal of Experimental Psychology: Animal Behavior Processes, 20, 390-401.

(Manuscript received December 5, 1997; revision accepted for publication May 6, 1998.) 